\title{
CSMAC: A New Centralized Scheduling-based MAC Protocol for Wireless LAN
}

\author{
Shunyuan Ye, Thanasis Korakis, Shivendra Panwar \\ Department of Electrical and Computer Engineering, Polytechnic Institute of NYU \\ e-mail: sye02@students.poly.edu, korakis@poly.edu,panwar@catt.poly.edu
}

\begin{abstract}
Medium Access Control (MAC) is a key issue for the efficiency of IEEE 802.11 wireless local area network. Legacy 802.11 DCF, using CSMA/CA and a random back-off mechanism, provides a simple-to-implement solution, however it achieves very low throughput. Exploiting the existence of a central entity in the infrastructure mode of an 802.11 wireless network, we propose a new centralized scheduling-based MAC protocol which is based on the popular DCF mechanism. This simple yet efficient scheme illustrates a new paradigm for realistic medium access control protocol design for next generation wireless networks. We have evaluated the performance of the proposed protocol by extensive simulations in different scenarios of wireless networks. Simulation results show that the new protocol significantly improves the network performance in terms of throughput and medium access delay.
\end{abstract}

Index Terms-Medium access control, IEEE 802.11, wireless network

\section{INTRODUCTION}

In recent years, WiFi networks based on 802.11 [1] have become very popular. Inexpensive access points make wireless Internet access a reality in corporations, campuses and homes. Almost all notebook computers today are equipped with an internal wireless interface. New generation handheld devices like PDAs and iPhones have access to WiFi networks. Although WiFi networks have become so popular and have dominated the market, their performance is not always good. This phenomenon is mainly because of the design characteristics of the MAC protocol of IEEE 802.11. An access mechanism that is based on contention in conjunction with an increase of the users in the same area lead to a performance that is far below optimal. Therefore, there is a need for a detailed investigation of the basic access protocol in order to improve its performance by applying simple yet efficient schemes on the top of the existing technology.

In the IEEE 802.11 protocol, the fundamental mechanism for accessing the medium is the Distributed Coordination Function (DCF). Under this mechanism, all stations share the medium using the Carrier Sense Multiple Access with Collision Avoidance (CSMA/CA) protocol and a random backoff mechanism. The standard also defines an optional Point Coordination Function (PCF), where a point coordinator determines which station has the right to transmit. Although PCF seems to be more effective for most applications, it was never commercialized due to several reasons. The main problem of $\mathrm{PCF}$ is its static character. It was designed as a polling scheme

This work is supported by by the New York State Center for Advanced Technology in Telecommunications (CATT), and the Wireless Internet Center for Advanced Technology (WICAT). that can only poll each station once during the contention free period. Therefore it cannot serve stations with higher traffic needs than others. On the other hand, since the access point gives to each station the chance to transmit in every polling circle, the existence of stations with sporadic traffic increases the overhead due to unnecessary polls. Finally, in order to enable the coexistence of PCF with DCF, 802.11 defines a superframe structure that consists of a contention free period (CFP) and contention period (CP). This structure significantly increase the implementation complexity, compared to DCF.

Therefore, DCF is the only scheme that is being used currently. Despite its popularity, DCF has some serious drawbacks. Since it was designed for ad-hoc communications, it does not take advantage of the existence of a centralized entity in infrastructure networks. Additionally, since it is based on contention, DCF suffers from collisions in a dense network. Bianchi [2] and Cali [3] have shown that DCF has a very poor throughput performance due to back-off and collisions.

Based on the above characteristics and limitations of the existing WiFi access methods, we designed a new MAC protocol that takes advantage of the existence of a centralized entity in infrastructure networks and optimizes the medium access control protocol. The proposed scheme is based on a simple yet efficient mechanism which is backward compatible with 802.11. In the new mechanism, the AP controls the medium access by adjusting the randomly generated back-off number of the stations. In this way the AP prevents collisions in the system, and in a way "schedules" the transmission of the existing stations. In saturation mode, when stations always have traffic to send, the new scheme can totally eliminate collisions and can achieve a throughput very close to the theoretical limit.

The rest of the paper is outlined as follows. In Section II, we give a brief description of DCF in 802.11 which is necessary for understanding the proposed protocol. In Section III, we describe the details of the mechanism that is used in the proposed scheme. The performance evaluation is carried out in Section IV and an extended set of results for networks with different parameters is presented. Section V completes the paper with our final conclusions and possible future work.

\section{IEEE 802.11 Medium ACCESS CONTROL}

This section briefly summarizes the DCF as defined by the IEEE 802.11 protocol. As illustrated in Figure 1, when a station has a new packet to transmit, it checks the channel activity using the carrier sensing mechanism. If the medium is idle for a period of time equal to Distributed InterFrame 


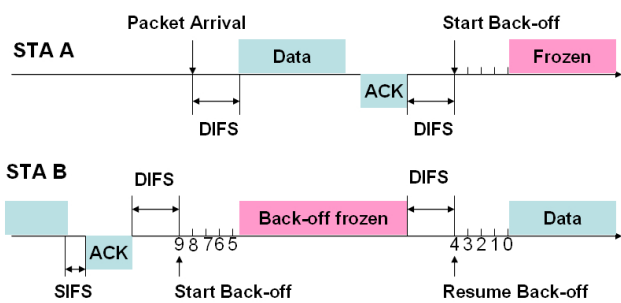

Fig. 1: 802.11 DCF

Space (DIFS), the station transmits. Otherwise, if the channel is sensed busy (either immediately or during the DIFS), the station will defer until the medium is idle for a DIFS period and then the back-off procedure will be invoked. Under this procedure, a station generates a random back-off number based on the contention window $(\mathrm{CW})$. The random back-off number is chosen from a uniform distribution over the interval $[0, \mathrm{CW}$ 1]. The station sets its back-off timer to be:

$$
\text { Backoff Time }=\text { Backoff Number } \times \text { aSlotTime }
$$

The back-off time counter decreases as long as the channel is idle, and stops decreasing when a transmission is detected on the channel. The countdown will start again when the channel is sensed idle for more than a DIFS. When the back-off counter reaches zero, the station starts its transmission.

The source station waits for an acknowledgment (ACK) immediately after a transmission. If it receives the ACK frame within the Short InterFrame Space (SIFS) period, the transmission is considered as successful and the contention window is reset to the initial value $C W_{\min }$. If the transmission fails, the contention window will be doubled. Sequential failures of transmission doubles again and again the $\mathrm{CW}$ until it reaches the maximum value $C W_{\max }$ (in the IEEE 802.11 DSSS specification, $C W_{\min }=31$ and $\left.C W_{\max }=1023\right)$. This process is called exponential back-off.

\section{The Proposed MAC Protocol}

\section{A. Motivation}

DCF uses CSMA/CA and a random back-off mechanism to access the medium. Both analysis and experimental results have shown that it has a very poor performance when the network is congested. Bandwidth is wasted for idle back-off time-slots and collisions. The network throughput is therefore far below the optimum. Bianchi [2] shows that, if all stations are using a distributed random access scheme, the optimum network throughput can be achieved when the transmission probability of each station is:

$$
\tau=\frac{1}{n \sqrt{T_{C}^{*} / 2}}
$$

Where $T_{C}^{*}=T_{C} / \sigma$ is the duration of a collision measured in slot time unit $\sigma$.

Based on this conclusion, several research efforts have been reported focusing on achieving a transmission probability for each station in the network close to the optimum value.

Some research [4], [5] in infrastructure networks, attempts to estimate how many stations are active in a Basic Service Set

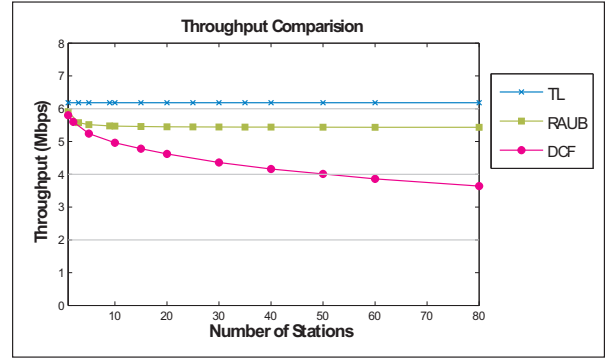

Fig. 2: Throughput Comparision

(BSS) and broadcast this information to all stations through the AP. The stations set their congestion window equal to $n \sqrt{T_{C}^{*} / 2}$, and therefore they achieve the optimum throughput. In [6], the authors derive the optimum number of idle time slots between two consecutive transmissions on the channel and propose a scheme that takes into consideration this information. Each station keeps sensing the medium. If the idle slots between two consecutive transmissions are more than the optimum value, the station decreases the congestion window, otherwise it increases it. Although the above mechanisms improve the existing medium access scheme, they have two significant drawbacks: 1) They increase the complexity of the medium access control mechanism 2)Their performance depends on the accuracy of their estimations. Additionally, even if they achieve the performance upper bound of random access schemes, their performance is still below the theoretical limit. In order to derive this theoretical limit we consider the following: suppose there is no idle slot or collisions. The channel is always busy due to successful transmissions. There is no propagation delay. Then the theoretical limit (TL) is:

$$
T L=\frac{\text { Packet Length }}{T_{D A T A}+T_{A C K}+T_{D I F S}+T_{S I F S}},
$$

where $T_{D A T A}$ is the time to transmit a packet, and $T_{A C K}$ the time for an acknowledge packet. The comparison of the theoretical limit, random access upper bound and DCF performance is shown in Figure 2.

Choi et al. [7] proposed a distributed reserved algorithm called EBA, which is similar to our scheme. Stations that have more packets in the buffer will try to reserve the next transmission slot by including their next transmission slot information in the current packet. Stations that receive and decode this packet will update their back-off counters to avoid collisions. EBA is a distributed algorithm and it does not utilize the existence of AP. It requires other stations to always decode all their neighbors' data packets, which is not feasible, especially when stations are using different data rates and they far away from each other. In our scheme, we overcome these difficulties by utilizing the existence of the AP. Stations only communicate with the AP and let the AP control the process. This approach is more efficient in WiFi networks that support multi-rate transmissions and therefore stations cannot always decode packets transmitted by neighbor stations.

In this paper, we propose a random access protocol that can achieve a performance very close to the theoretical limit, and exceeds the upper bound of the random access schemes. The main feature of our protocol is that although it is a random 
access scheme, it takes advantage of the existence of the AP in an infrastructure network. Under this new scheme, when a station has more than one packet to send, it generates the back-off number for the second packet before sending the first one. This back-off number is included in the header of the first packet in order to inform the AP about the intention of the station to transmit another packet in the near future. The AP keeps a list of the slots that have been reserved by other stations. If the intending transmission does not collide with other reserved slots, this back-off number will be confirmed by the AP though the ACK of the current packet. Otherwise, the AP will generate another back-off number and will return it to the station through the ACK. Based on this scheme, collisions are significantly reduced since now there is a centralized entity that schedules the transmissions. In case that all stations have always packets to send, after a very short time, there are no collisions in the system since every station reserves the appropriate time to transmit its next packet.

\section{B. Proposed MAC Protocol}

The proposed MAC protocol is based on the DCF of IEEE 802.11. As we mentioned the protocol does not change the access method, it only adjusts the back-off number of each station. The details of the protocol are as follows:

1) Step One, Station Behavior: A station that intends to transmit a packet checks its transmission queue. If there is more than one packet in the queue, the station will normally invoke the back-off procedure after the first packet's transmission, in order to proceed with the next one. Instead of doing this, the station will generate a random back-off number (for the second packet) before the transmission of the first packet. If the current transmission is successful, this back-off number will be used for the transmission of the next packet. This information is included in the header of the current packet, in a new subfield called next back-off number and is sent to the AP. In the case that there is only one packet in the queue, there will be no back-off after the current transmission. In this case, the next back-off number subfield will be set to -1 in the header of the packet.

2) Step Two, AP Behavior: The AP keeps a list of the stations and the time-slots that are already reserved for the next transmissions. Once a new packet received, the AP checks the next back-off number field in the packet and looks up the list. If that time-slot is not reserved yet, the AP will add a new entry into the list. Otherwise, the AP will generate a new back-off number for the specific station. An obvious way to pick this time-slot is to choose any available slot. However we propose a specific algorithm for this selection. Under this, the new back-off number generation is based on the formula below:

$$
\text { New Backoff }=\text { Last Reserved }+ \text { Random }[1,8]
$$

Using this algorithm, the reserved time-slots are spaced out. This can reduce the collisions for stations that have not reserved time-slots for their packet transmissions yet.

We should mention here that the "time-slot" that is reserved for the transmission of a particular packet is the slot that

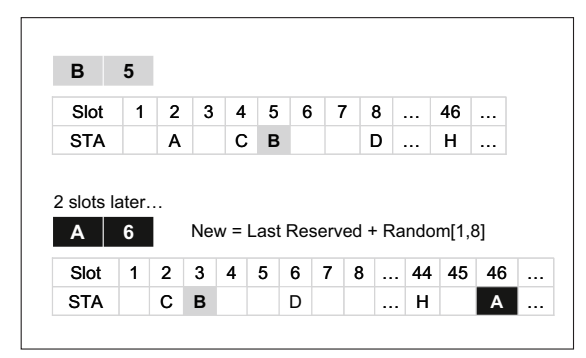

Fig. 3: Time-slot Reservation List

indicates the beginning of the transmission. During the transmission, both the AP and the stations "freeze" their back-off counters and therefore the process is independent of the packet size.

3) Step Three, Station Behavior: On receiving the ACK from the AP, the station checks the next back-off number that was returned by the AP. If the number is the same as the one initially defined by the station, this is an indication that there were no reservations for that particular time-slot. Therefore, the station will set the congestion window to be $C W_{\text {min }}$, and start the back-off procedure. Otherwise, if the back-off number has already been changed by the AP, this is defined as a "virtual collision". The station will double its congestion window and use the new back-off value to start the back-off procedure.

Here we should point out that in the proposed scheme, the AP does not totally control the scheduling in the system. Stations make the reservations. The AP just adjusts those reservations in a way that it will avoid collisions. The reason why we do not allow the AP to schedule the transmissions for the stations is that the system is dynamic in the sense that not all the stations are always "scheduled". There may be newly active stations which have not reserve their next transmission slot yet. Therefore, there is a probability that scheduled stations may collide with unscheduled ones. After the collision, they have to generate the back-off numbers by themselves, using their contention window. This is more efficient than a static schedule generated by the AP since scheduled stations adjust their contention windows based on the particular reservation and therefore they dynamically adjust their next packet transmission.

4) An illustration: In order to describe the basic functionality of the scheme we give the following example. We call stations which have already reserved slots for their next transmissions "scheduled" and those that have not reserved slots yet "unscheduled".

The list maintained by the AP is shown in Figure 3. Initially, the AP receives a packet from STA $B$, which tries to reserve the time-slot 5 for future packet transmission. Since this timeslot is not reserved yet, the AP adds this reservation into the list, and returns the number 5 back to STA B. As long as the channel is idle, AP decreases the time-slot numbers in the list. Two time-slots later, STA A sends a packet to AP, trying to reserve slot 6 . This time-slot has been already reserved by STA D, so AP generates a new back-off number using (4). The last reserved time-slot is 44 , and the random number is 2 , so the new back-off number is 46 . This number is returned back to STA A. 


\section{Analysis}

The proposed MAC protocol allows the stations to reserve a time-slot for the next packet transmission. This scheme works only when there are more than one packets in the transmission queue. If we model the transmission queue of a station in the network to be an $M / M / 1$ queue, the probability that our new protocol can derive some gain is the probability that the queue has more than one packet. This probability can be written as the formula below:

$$
P_{\text {Gain }}=1-P_{0}-P_{1}=1-(1-\rho)-(1-\rho) \rho=\rho^{2}
$$

where $P_{0}$ is the probability of an empty queue, $P_{1}$ is the probability that only one packet is in the queue and $\rho$ is the utilization. As the network becomes more and more congested, our protocol has a higher and higher probability to obtain a gain as $\rho$ increases.

\section{Revision}

The proposed protocol eliminates the collisions among scheduled stations. However, it cannot avoid the collisions caused by unscheduled stations. When an unscheduled station has a new packet to send, it starts transmitting right away or it generates a random number to start the back-off procedure, depending on the current channel activity. If an unscheduled station transmits a packet at a slot that is reserved by a scheduled station, a collision occurs. As an additional sideeffect, the scheduled station becomes unscheduled. This "new" unscheduled station may collide with another scheduled station again and make it unscheduled. This may lead to more and more unscheduled stations in the network, and increase the numbers of collisions.

In order to see the effect of this phenomenon in the performance of the scheme, we consider a scenario in which there are $\mathrm{N}$ stations in the network. Initially, all stations are unscheduled. Each station is in saturation mode and therefore, it always has packets in its queue waiting for transmission. We can model this scenario as a Markov Chain. The state is defined as the number of scheduled stations in the network. When the model goes to state $\mathrm{N}$, all $\mathrm{N}$ stations are scheduled, and their transmission slots are all reserved from AP. Therefore, there are no collisions in the system. We define this status as "Convergence". Initially the system starts from state 0 and it converges after some time. The converge time may be short, or may go to infinity, depending on the number of the stations in the network.

In particular, the phenomenon we described above, generated as unscheduled stations collide with scheduled stations, may increase the convergence time. Every time a station transmits a packet, it becomes scheduled (and the Markov chain state increases by one). However, when a collision occurs between an unscheduled and a scheduled station, the scheduled station becomes again unscheduled and therefore the chain status decreases by one. In order to cancel this inefficiency and reduce the convergence time, we revise the protocol as follows:

1) AP Behavior: The back-off time-slots are divided into two groups, odd and even. When a station tries to reserve a

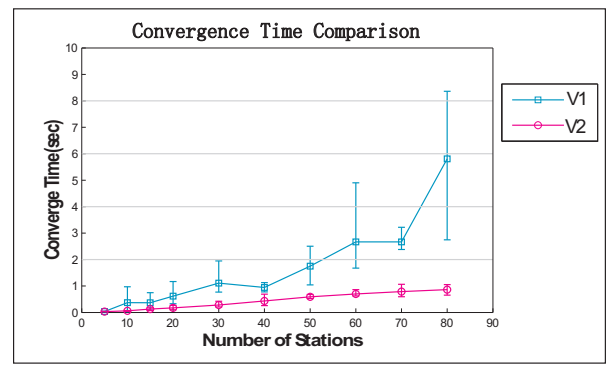

Fig. 4: Convergence Time

time-slot for its next transmission, the AP adjusts it to even slots only.

2) Scheduled Station: When generating the random number for the next back-off, a scheduled station only generates even numbers. On the other hand, when this station collides with unscheduled stations (and becomes unscheduled), it generates an odd number for back-off. Using this differentiation, a scheduled station that becomes unscheduled again will not collide with other scheduled stations when it starts retransmitting.

3) Unscheduled Station: Initially, when there is a new packet for transmission, an unscheduled station senses the channel. If it is busy, the station generates a random number and starts the back-off process. Since this back-off number is totally random, the unscheduled station may collide with other stations, both scheduled and unscheduled. In order to avoid the collision with scheduled stations, this unscheduled station keeps listening to the channel. Every time it receives an ACK (transmitted by the AP to some other station) it checks the next back-off number field in the header. If it is not -1 , then that slot is scheduled for the next transmission of a scheduled station. The unscheduled station can then differentiate whether its next transmission slot is in the same group of slots as the scheduled stations or not. It will adjust its back-off number if it is in the same group of slots as the scheduled stations. By doing this, the unscheduled stations will use different group of slots than the scheduled stations and therefore they will avoid collisions.

In order to evaluate the efficiency of this scheme we ran several simulations for different numbers of stations in the network. The results are shown in Figure 4. V1 is the initial version of the protocol while V2 is the version that includes the above scheme. In each scenario we ran 5 simulations. In the figure we present the average, the maximum and the minimum of the convergence time for both versions. As we can see, it takes very short time for the revised version to converge. Even in a large network of 80 stations, the convergence time is less than 1 second. Additionally, the revised version has a much smaller variance in the convergence time.

\section{PERformance Evaluation}

In order to evaluate the performance of the proposed protocol and gain deeper understanding of the protocol behavior in a large scale network, extensive simulations have been conducted. For the simulations we used OPNET. We present simulation results below where we compare our protocol to IEEE 802.11. 


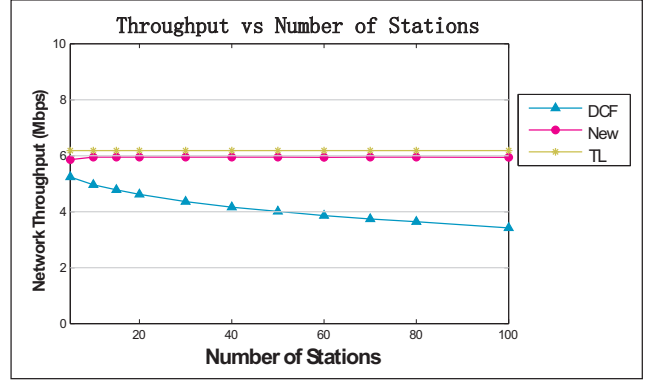

Fig. 5: Throughput vs Number of Stations

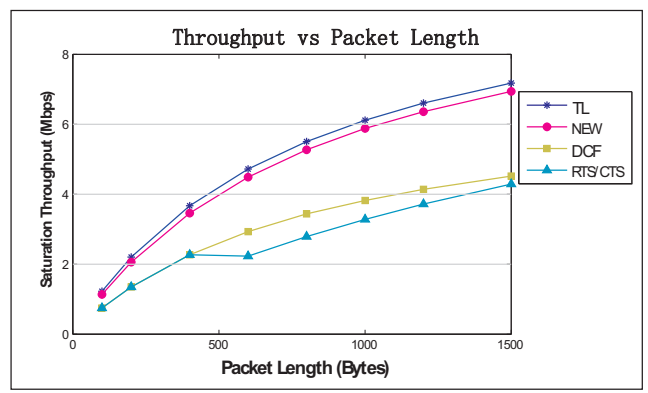

Fig. 6: Throughput vs Packet Length

\section{A. Simulation Settings}

In order to simulate the new proposed MAC protocol we modified the OPNET model of IEEE 802.11. Each station in the network communicates directly with the AP at a data rate of $11 \mathrm{Mbps}$. Each station in the network transmits packets to the AP. For each scenario we collected the aggregate network throughput and the service delay. The data presented hereafter was averaged over 20 runs, each of which used a different random initial seed and ran for 600 seconds which was long enough to get stabilized results.

\section{B. Comparison with DCF}

Figure 5 reveals the relation between the network throughput and the number of nodes deployed. The MSDU packet size is 1024 bytes. To obtain the system capacity, the network is saturated and each node is in a backlogged state. It is apparent that the new proposed MAC scheme achieves a throughput which is very close to the theoretical limit (TL), and outperforms 802.11 DCF.

The fact that our proposed medium access control scheme is very close to the theoretical limit is due to the following reasons. As all stations in the network are saturated, they keep reserving the time-slots for their next packet transmission. Therefore, once convergence is achieved, the system has no

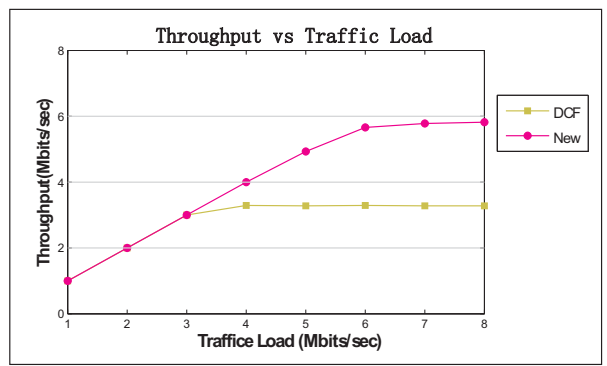

Fig. 7: Throughput vs Traffic Load

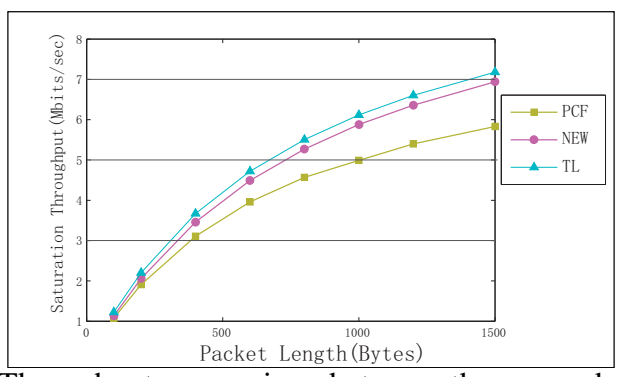

Fig. 8: Throughput comparison between the new scheme and PCF in saturation load conditions

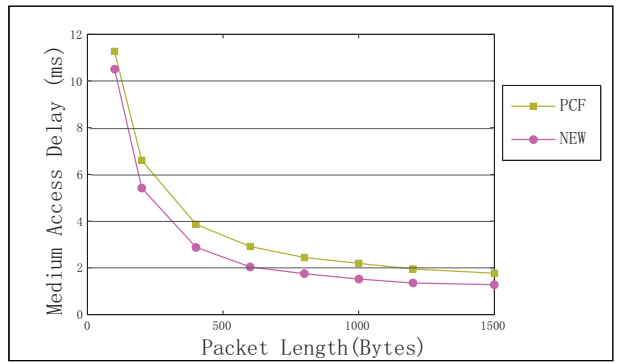

Fig. 9: Delay comparison between the new scheme and PCF in saturation load conditions

collisions any more. The only wasted resource is some idle back-off slots, which can reduce the collisions and medium access delay for unscheduled stations that have new packets to send.

In the second part of our simulations we ran scenarios with different packet sizes and compare the performance of our proposed protocol with DCF, with and without RTS/CTS. The threshold to turn on RTS/CTS is set to 500 bytes. Figure 6 shows that the new MAC protocol delivers higher throughput than legacy 802.11 DCF. In the case that RTS/CTS is used, the 802.11 throughput decreases. This is due to the fact that the data rate of the transmissions is $11 \mathrm{Mbps}$. With these high rates, the transmission time is very short and therefore the RTS/CTS mechanism adds overhead into the network instead of improving the performance.

In the next simulation, we consider scenarios with different traffic loads in the network with 60 stations. The packet size is 1024 Bytes. Figure 7 shows that the proposed MAC protocol has higher network capacity and thus can handle much heavier traffic load than the legacy DCF.

\section{Comparison with PCF}

The above results have shown that the new MAC protocol has better performance than legacy $802.11 \mathrm{DCF}$, due to the involvement of the AP in the medium access control. In IEEE 802.11 there is another access scheme called Point Coordination Function (PCF). PCF is based on a polling mechanism that sequentially polls each station in the network. In order to further evaluate our protocol, we compare it with PCF. We first consider saturation mode for both schemes. Stations always have packets to send. Figure 8 and 9 show the throughput and delay comparisons. CSMAC performs better since it can schedule transmissions without any polling overhead.

We then consider a scenario where stations generate traffic with exponential $\mathrm{ON}$ and $\mathrm{OFF}$ periods. Figure 10 reveals that 


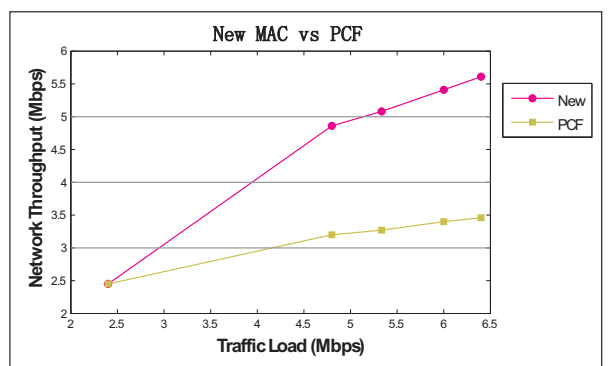

Fig. 10: Throughput: CSMAC vs Dynamic PCF

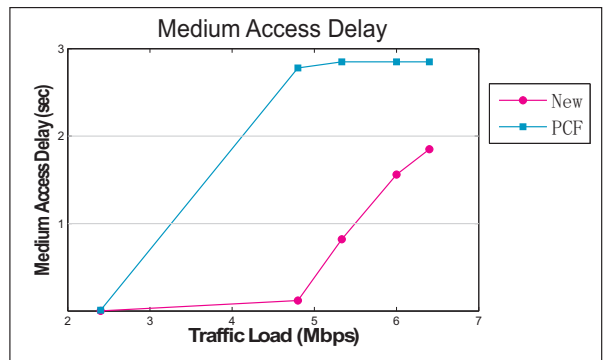

Fig. 11: Delay: CSMAC vs Dynamic PCF

the new MAC protocol has even higher throughput than PCF. This is because in PCF the AP keeps polling the stations in order to give them a chance to transmit. However, if the transmission queue of the polled station is empty, the transmission period of the polling packet is wasted. In order to add a "dynamic" character to PCF, we implemented PCF using a scheme where if the polled station does not transmit any packet in two consecutive super-frames, that station will be removed from the polling list of the AP. As we can see in the results, even this "dynamic" polling scheme has a worst performance than our protocol. In the new MAC protocol, the AP does not have to maintain a polling list. It schedules the transmissions of the stations "on-demand" based on their needs for the next packet transmission.

We compare the medium access delay performance of the above scenario in Figure 11. As we can see, our MAC scheme outperforms 802.11 PCF even for delay performance. This is due to the fact that when a station is removed from the polling list and generates a new packet to send, it has to wait until the start of the contention period (CP) of the particular super-frame where it has to content for the channel to get again the chance to be polled. Therefore, it takes much more time for this station to send the packet to the AP and become again a "polling station". However, in our protocol there is not such a waist of time since there is no differentiation between a contention free and a contention period. Therefore, our protocol is much more adaptive to the dynamic traffic changes of the stations.

\section{VoIP Performance}

An interesting study for the performance of our scheme would be the examination of its behavior when VoIP traffic is active in the network. Therefore, we setup a series of simulations in order to evaluate the scheme in such conditions. The traffic of each connection is as in [8], 160 Bytes per 20 $\mathrm{ms}$, on both up-link and downlink traffic. Figure 12 depicts

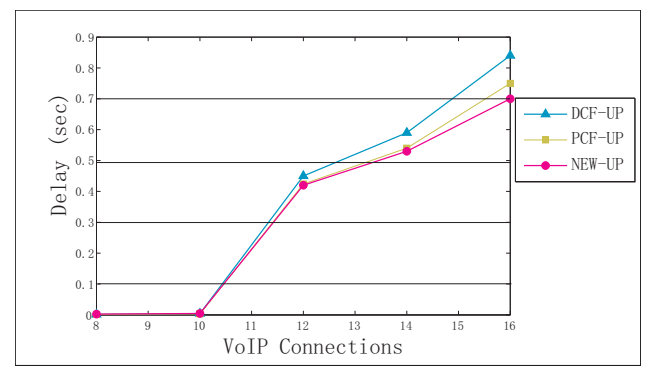

Fig. 12: VoIP Delay Comparison

the delay performance for the uplink channel for the three different schemes. As we can see, CSMAC has a lower delay than DCF and PCF. Therefore, given a delay budget, CSMAC can allow for more simultaneous VoIP connections than the other schemes.

\section{CONCLUSIONS}

In this paper we propose and study a new medium access control protocol for 802.11 wireless networks. In this protocol, the AP helps in the scheduling of the transmissions of the stations by maintaining a list of the time-slots that have been reserved for their next transmissions. This schedule-based distributed access scheme leads to a significant reduction of collisions in the network. Since the nature of the scheme is dynamic, it adapts its behavior in the changes of the network resulting to a simple yet very efficient access mechanism. We studied the performance of the proposed scheme using simulation results for different scenarios and different network settings. We compared the new mechanism with legacy IEEE 802.11 DCF and PCF. The study shows that the new MAC protocol significantly outperforms DCF and PCF due to the fact that it is adaptive and thus more efficient. The new scheme is very easy to implement. Based on the promising results as well as on the simplicity of the protocol we believe that the new scheme illustrates a new design paradigm for realistic medium access control for next generation wireless networks.

\section{REFERENCES}

[1] "Part 11: Wireless LAN Medium Access Control (MAC) and Physical Layer (PHY) Specifications," ANSI/IEEE Std 802.11, 1999 Edition, 1999.

[2] G. Bianchi, "Performance analysis of the IEEE 802.11 distributed coordinationfunction," IEEE Journal on Selected Areas in Communications, vol. 18, pp. 535-547, March 2000.

[3] F. Cali, "Dynamic tuning of the IEEE 802.11 protocol to achieve a theoreticalthroughput limit," IEEE/ACM Transactions on Networking, vol. 8, pp. 785-799, December 2000.

[4] G. Bianchi and I. Tinnirello, "Kalman filter estimation of the number of competing terminals in an IEEE 802.11 network," in Proceedings of INFOCOM'03, (San Francisco, USA), March 2003.

[5] H. Ma, H. Li, P. Zhang, S. Luo, C. Yuan, and X. Li, "Dynamic Optimization of IEEE 802.11 CSMA/CA Based on the Number of Competing Stations," in Proceedings of ICC'04, (Paris, France), June 2004.

[6] M. Heusse, F. Rousseau, R. Guillier, and A. Duda, "Idle Sense: An Optimal Access Method for High Throughput and Fairness in Rate Diverse Wireless LANs," in Proceedings of ACM Sigcomm'05, (Pennsylvania, USA), August 2005.

[7] J. Choi, J. Yoo, S. Choi, and C. Kim, "EBA: An Enhancement of the IEEE 802.11 DCF via Distributed Reservation," IEEE Transactions on Mobile Computing, vol. 4, July 2005.

[8] W. Wang, S. Chang, L. Li, and V. Li, "Solutions to performance problems in VoIP over a 802.11 wireless LAN," IEEE Transactions on Vehicular Technology, vol. 54, pp. 366-384, Jan 2005. 